\title{
Spinal cord infarction with resultant paraplegia after Chiari I decompression: case report
}

\author{
Amar S. Shah, MD, ${ }^{1}$ Alexander T. Yahanda, MS, ${ }^{1}$ Umeshkumar Athiraman, MD, ${ }^{2}$ \\ Rene Tempelhoff, MD, ${ }^{2}$ and Michael R. Chicoine, MD' \\ Departments of ${ }^{1}$ Neurosurgery and ${ }^{2}$ Anesthesia, Washington University School of Medicine, St. Louis, Missouri
}

Paraplegia after posterior fossa surgery is a rare and devastating complication. The authors reviewed a case of paraplegia following Chiari decompression and surveyed the literature to identify strategies to reduce the occurrence of such events.

An obese 44-year-old woman had progressive left arm pain, weakness, and numbness and tussive headaches. MRI studies revealed a Chiari I malformation and a cervicothoracic syrinx. Immediately postoperatively after Chiari decompression the patient was paraplegic, with a T6 sensory level bilaterally. MRI studies revealed equivocal findings of epidural hematoma at the site of the Chiari decompression and in the upper thoracic region. Surgical exploration of the Chiari decompression site and upper thoracic laminectomies identified possible venous engorgement, but no hematoma. Subsequent imaging suggested a thoracic spinal cord infarction. Possible explanations for the spinal cord deficit included spinal cord ischemia related to venous engorgement from prolonged prone positioning in an obese patient in the chin-tucked position. At 6.5 years after surgery the patient had unchanged fixed motor and sensory deficits.

Spinal cord infarction is rare after Chiari decompression, but the risk for this complication may be increased for obese patients positioned prone for extended periods of time. Standard precautions may be insufficient and intraoperative electrophysiological monitoring may need to be considered in these patients.

https://thejns.org/doi/abs/10.3171/2019.10.SPINE19921

KEYWORDS Chiari malformation; posterior fossa decompression; paraplegia; complication; neuromonitoring; congenital

$\mathrm{T}$ $\checkmark$ HE Chiari I malformation is a congenital disorder that is characterized by a low-lying position of the cerebellar tonsils and may be associated with a spinal cord syrinx., ${ }^{9,24,34,37}$ Surgical treatment for symptomatic Chiari malformations with tussive headaches or other neurological symptoms involves a midline suboccipital craniectomy with or without cervical laminectomies. Treatment variations include extradural decompression with lysis of the ligamentous bands at the foramen magnum and dural opening with dural patch grafts of various types. ${ }^{7,10,23,27,28,38,44}$ Intraoperative ultrasound ${ }^{12,31,32}$ and intraoperative MRI (iMRI) ${ }^{6}$ have been used to help the surgeon decide whether an extradural decompression is adequate or whether a dural opening and patch graft should be considered. Other potential adjunct maneuvers include wide opening of the subarachnoid spaces and coagulation of the cerebellar tonsils. ${ }^{16,35}$ These are established neurosurgical techniques with generally low rates of neurological complications, but there are nevertheless risks of debilitating or fatal complications.

\section{Case Report}

A morbidly obese 44-year-old woman (BMI $45 \mathrm{~kg} / \mathrm{m}^{2}$, height 63 inches [160 cm], weight $115.6 \mathrm{~kg}$ ) with progressive left arm pain, weakness, and numbness and tussive headaches had previously been diagnosed with a degenerative shoulder disorder. Her past medical history included hypertension controlled with 2 medications, prior hysterectomy, and a history of cigarette smoking with occasional alcohol and marijuana use. Her ASA (American Society of Anesthesiologists) score ${ }^{40}$ was 3 . An MRI study revealed a Chiari I malformation with $19 \mathrm{~mm}$ of cerebellar tonsillar descent below the foramen magnum and a cervicothoracic syrinx from the second cervical spinal level (C2) to the fifth thoracic spinal level (T5) (Fig. 1A-C), but

ABBREVIATIONS iMRI $=$ intraoperative MRI. 
Shah et al.
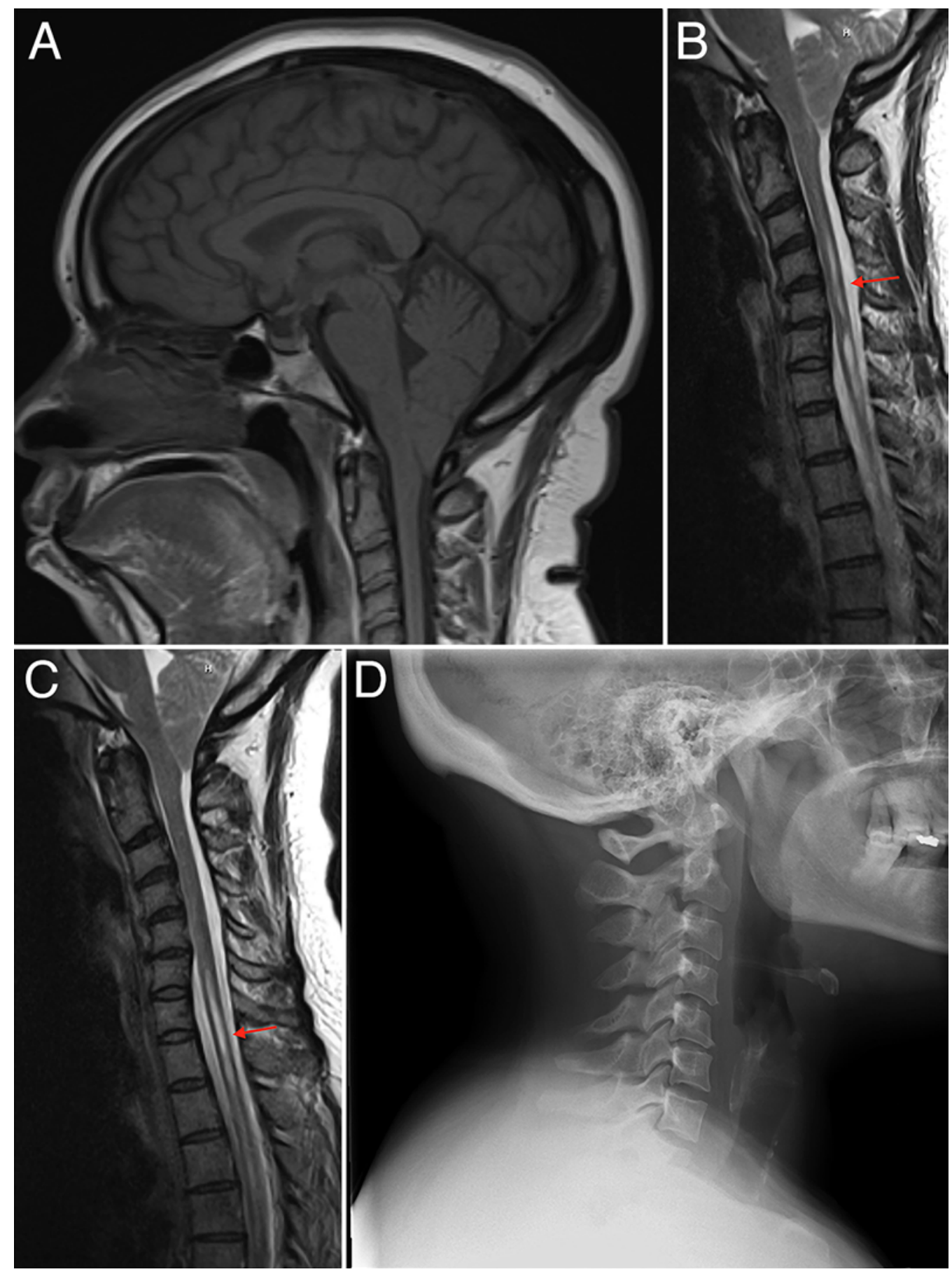

FIG. 1. Preoperative imaging demonstrating Chiari I malformation in a T1-weighted sagittal MR image of the head (A), cervicothoracic syrinx (red arrows) in T2-weighted sagittal MR images of the cervical (B) and upper thoracic (C) spine, and degenerative cervical spine findings in a plain lateral cervical spine radiograph (D). Figure is available in color online only.

no hydrocephalus or other anomalies. Additional inferior thoracic and lumbar spine imaging was not obtained. Plain radiographs of the cervical spine, including flexion and extension views, showed only mild degenerative disease at C5-6 (Fig. 1D). The radiologists concluded that the patient's degenerative shoulder condition was likely a Charcot joint ${ }^{5,13}$ associated with her syrinx (Fig. 2).
Due to the patient's symptoms, Chiari decompression was recommended. The routine preoperative assessment documented normal sinus rhythm, normal blood pressure, and a normal chest radiograph. In light of the syrinx, a fiber-optic bronchoscopy was performed with endotracheal intubation to minimize cervical spine manipulation. The Chiari decompression was performed in an iMRI 
suite (IMRIS, Inc.) according to a research protocol in which iMRI would be used during the surgery to assess the adequacy of the decompression. The patient was positioned prone on the iMRI-compatible operating table with an iMRI-compatible 3-pin head holder and the head in a standard chin-tucked position to maximize access to the posterior fossa and upper cervical region. Padded chest rolls were utilized to minimize intraabdominal pressure. The prone positioning was particularly challenging due to the narrow width of the iMRI-compatible operating table and the patient's large body habitus.

Initially, a midline suboccipital craniotomy, $\mathrm{C} 1$ laminectomy, and sectioning of the thickened dural bands and ligaments were performed to achieve an extradural Chiari decompression. An iMRI (Fig. 3 left) obtained after completion of the extradural decompression suggested incomplete decompression and poor CSF flow on the cine flow sequences. These findings prompted the use of subsequent dural opening and a patch graft to achieve a more expansive decompression, after which another iMRI was completed (Fig. 3 right). Because the case was made more complex by the patient's BMI, positioning challenges, fiber-optic intubation, intravenous and intraarterial access, the 2-part procedure with the extradural and intradural components, and the iMRI studies, the duration of the surgery was 4 hours and 40 minutes. The patient's cardiopulmonary parameters remained stable throughout the procedure. Her arterial blood pressures ranged from 90 to $140 \mathrm{~mm} \mathrm{Hg}$ systolic and 50 to $60 \mathrm{~mm} \mathrm{Hg}$ diastolic, her heart rate was 80 to 100 beats per minute, and oxygen saturations were greater than $95 \%$. Peak airway pressures were modestly elevated in the range of 35 to $40 \mathrm{~cm}$ of water, consistent with the patient's obesity and the prone position. The estimated blood loss was $200 \mathrm{ml}, 4 \mathrm{~L}$ of intravenous crystalloids were administered intraoperatively, and $250 \mathrm{ml}$ of urine output was recorded.

Upon preparation for extubation and awakening at the completion of the operation, the patient was able to move her upper extremities with full strength but was completely paraplegic with no ability to move or sense her lower extremities. Review of the iMR images showed no conclusive explanation for this new-onset paraplegia. Urgent MRI studies of the occipital, cervical, thoracic, and lumbar regions were obtained in the neuroradiology department, as the iMRI device could not image below the midcervical region. These additional MRI studies revealed equivocal evidence of an epidural hematoma in the occipital-cervical region at the site of the Chiari decompression and in the upper (T3-7) thoracic region (Fig. 4). The patient was returned to the operating room emergently for both exploration of her thoracic spine with laminectomy at $\mathrm{T} 3$ and exploration of the occipital-cervical site of her Chiari decompression. Neither exploration identified an epidural hematoma or other obvious etiology of her paraplegia. A considerable amount of highly vascularized epidural adipose tissue with venous engorgement was present in the upper thoracic epidural space, which was treated with bipolar cauterization to achieve hemostasis and to reduce the volume of epidural adipose tissue.

After the second operation, there was no improvement in the patient's condition, and she remained paraplegic

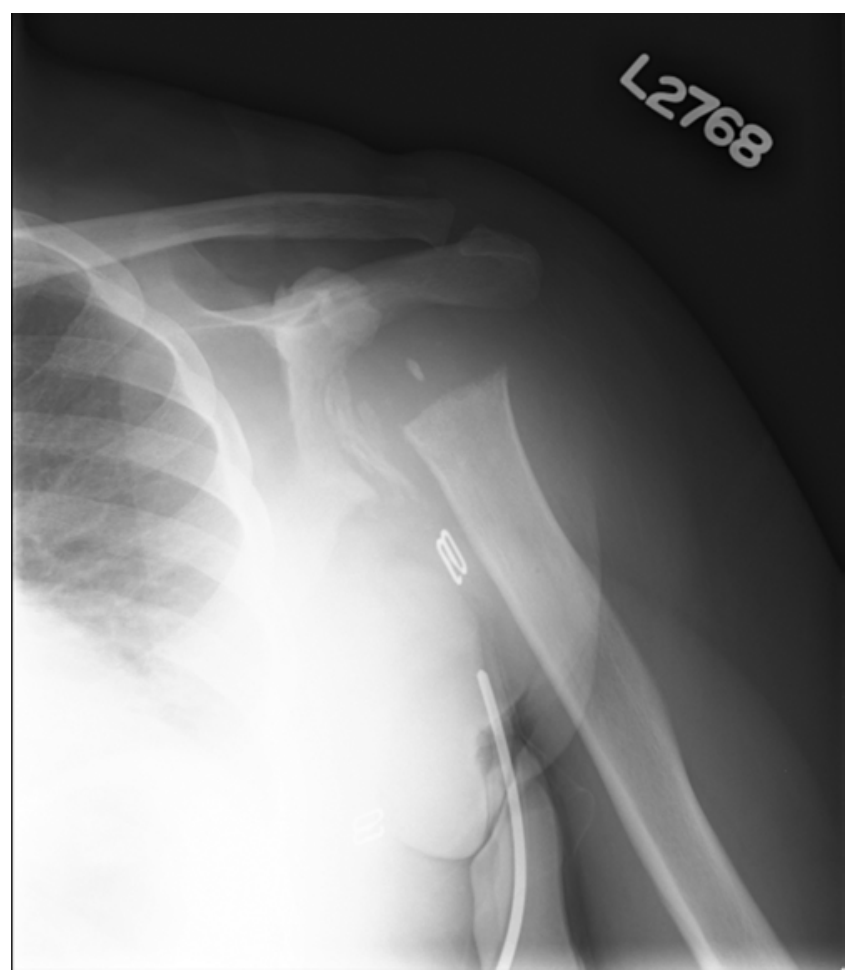

FIG. 2. Preoperative radiograph of the left shoulder demonstrating absence of the left humeral head and remodeling of the left glenoid fossa, likely secondary to a neuropathic Charcot joint.

with a midthoracic sensory level. High-dose steroids were initiated, given the uncertain etiology of a suspected spinal cord injury, as per National Acute Spinal Cord Injury Study protocol. ${ }^{8}$ (If this situation were to occur today, high-dose steroids may not be administered, but this case occurred in 2009, at which time there was still ongoing debate about the role of steroids in spinal cord injury.) The patient received a bolus dose of $3600 \mathrm{mg}$ methylprednisolone and was placed on a continuous intravenous infusion of $647 \mathrm{mg}$ methylprednisolone per hour $(5.4 \mathrm{mg} / \mathrm{kg} / \mathrm{hr})$ for 23 hours. She did not regain voluntary movement of her lower extremities. Repeat MRI 7 days after surgery demonstrated findings suggestive of a spinal cord infarct from T3 to T6 (with abnormal signal from T2 to T10) (Fig. 5 ) and possibly some residual venous engorgement. Additional spinal imaging was recommended in the weeks after surgery, but the patient declined all imaging. She was discharged to an inpatient rehabilitation facility 9 days after surgery. At the time of last assessment 6.5 years after surgery, the patient's paraplegia and other impairments had persisted with minimal recovery. She has remained wheelchair bound and required suprapubic catheter placement, a diverting colostomy, and multiple wound debridements for decubitus ulcers.

\section{Discussion}

Potential complications after posterior fossa decompression for Chiari malformations include pseudomeningocele, CSF leak, hydrocephalus, meningitis, headache, 

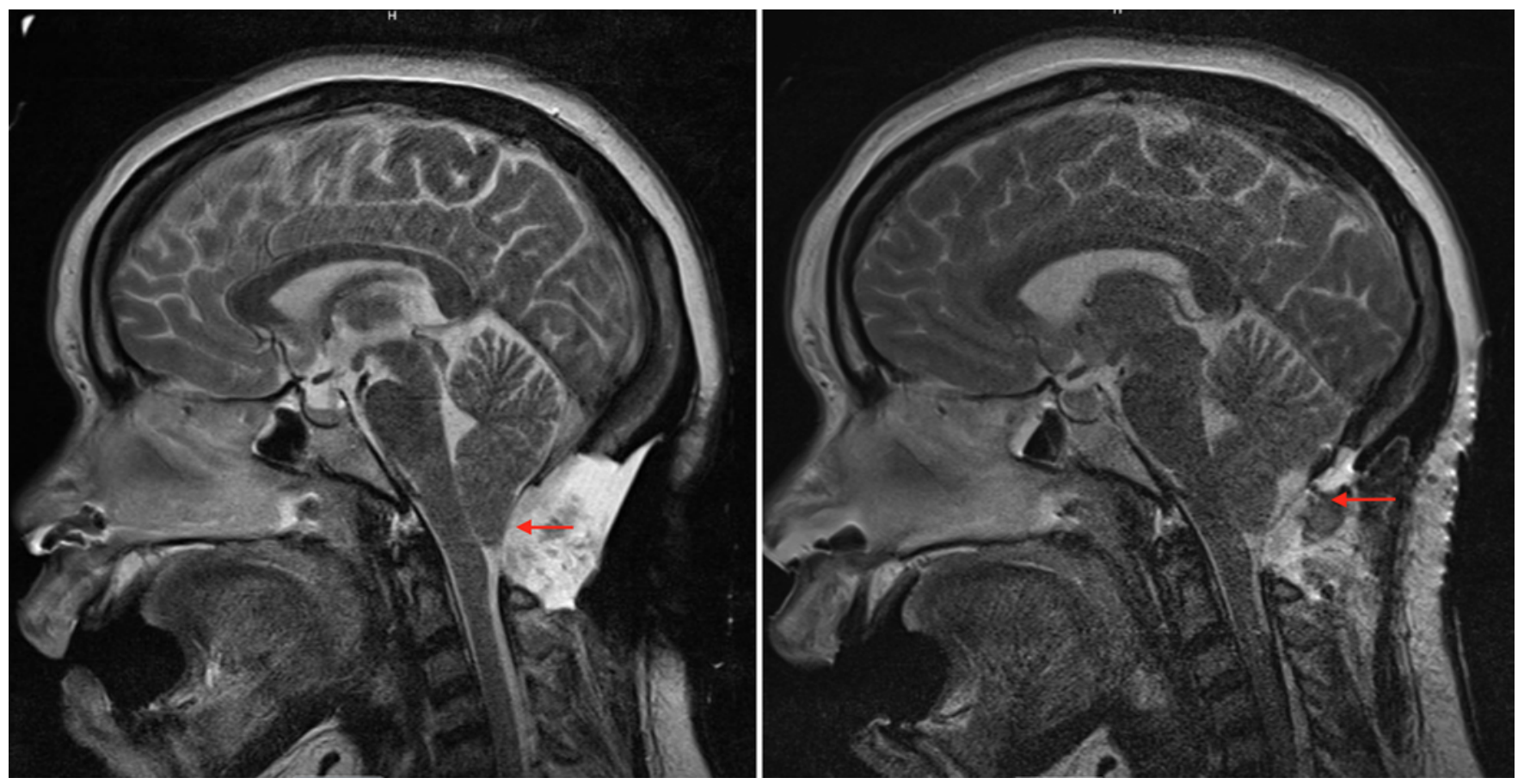

FIG. 3. Intraoperative T2-weighted sagittal MR images of the head and upper cervical spine obtained after extradural compression, which demonstrate some potential residual tonsillar compression (left, red arrow), and after duraplasty, which demonstrate a more generous CSF space dorsally at the cervicomedullary junction (right, red arrow). Figure is available in color online only.

wound complications, and failure of symptoms to improve. ${ }^{45}$ Spinal cord infarction after posterior fossa surgery is a rare event. Characterization of factors that may increase the risk of this devastating phenomenon is important to minimize its occurrence. In order to better clarify these risk factors, we analyzed the current case of paraplegia after Chiari I decompression in an obese woman and reviewed the relevant published literature.

Subdural and epidural hematomas are potential causes of postoperative neurological deficits after cranial and spinal surgery. However, in this particular case, surgical exploration of the occipital-cervical site of the Chiari decompression and of the upper thoracic region failed to identify any significant hematoma. Additionally, the MRI findings of increased T2 signal in the midthoracic region were concerning for spinal cord edema or infarction, though flow artifacts in imaging of the thoracic spine admittedly can make such imaging challenging. ${ }^{14}$ There are 3 cited potential causes of spinal cord infarction ${ }^{29,33}$ following posterior fossa operations: 1) cervical hyperflexion leading to spinal cord lengthening and hypoperfusion, 2) compression of the vasocorona secondary to rapid CSF loss from the cisterna magna leading to a pressure differential between medullary and infratentorial compartments, and 3) spinal venous congestion due to increased intraabdominal pressure from positioning. Each of these causes may have been a contributing factor in the current case. The patient's neck was flexed for the duration of the operation, as is necessary to expose the midline occipital-cervical region for a Chiari decompression (particularly for an obese individual), and this sustained neck flexion may have predisposed her to spinal cord hypoperfusion. Intracranial hypotension has been associated with epidural venous enlargement, ${ }^{11,42}$ and in one reported case intracranial hypotension due to a dural opening was hypothesized to have resulted in spinal epidural venous engorgement leading to cord compression. ${ }^{1}$ Rare conditions such as Hirayama disease, a monomelic amyotrophy associated with cervical flexion, ${ }^{17}$ and surfer's myelopathy, a nontraumatic myelopathy associated with cervical extension, ${ }^{18}$ are other conditions in which neurological deficits occur secondary to spinal cord hypoperfusion.

The patient's morbid obesity and the protracted nature of the surgery due to challenges with her airway and positioning, along with the added time required for the iMRI study, likely led to a prolonged period of increased intraabdominal pressure and spinal cord ischemia. Increased intraabdominal pressure can lead to increased venous pressure in the inferior vena cava, decreased venous return, reduced cardiac output, increased bleeding, and venous stasis. ${ }^{15}$ Abdominal compression and mild hypoventilation have been estimated to reduce the spinal subarachnoid space by up to $40 \% .{ }^{25}$ Spinal epidural venous plexus engorgement has been reported in patients with increased intraabdominal pressure from pregnancy and obesity. ${ }^{21}$ Notably, in the patient reported here, the operative findings at the time of the second operation did include a qualitative sense of venous engorgement of the epidural plexus, but the anesthesia team did not detect any aberrations on monitoring that led them to suspect abnormal intraabdominal pressure that could have compromised blood flow to the spinal cord. 

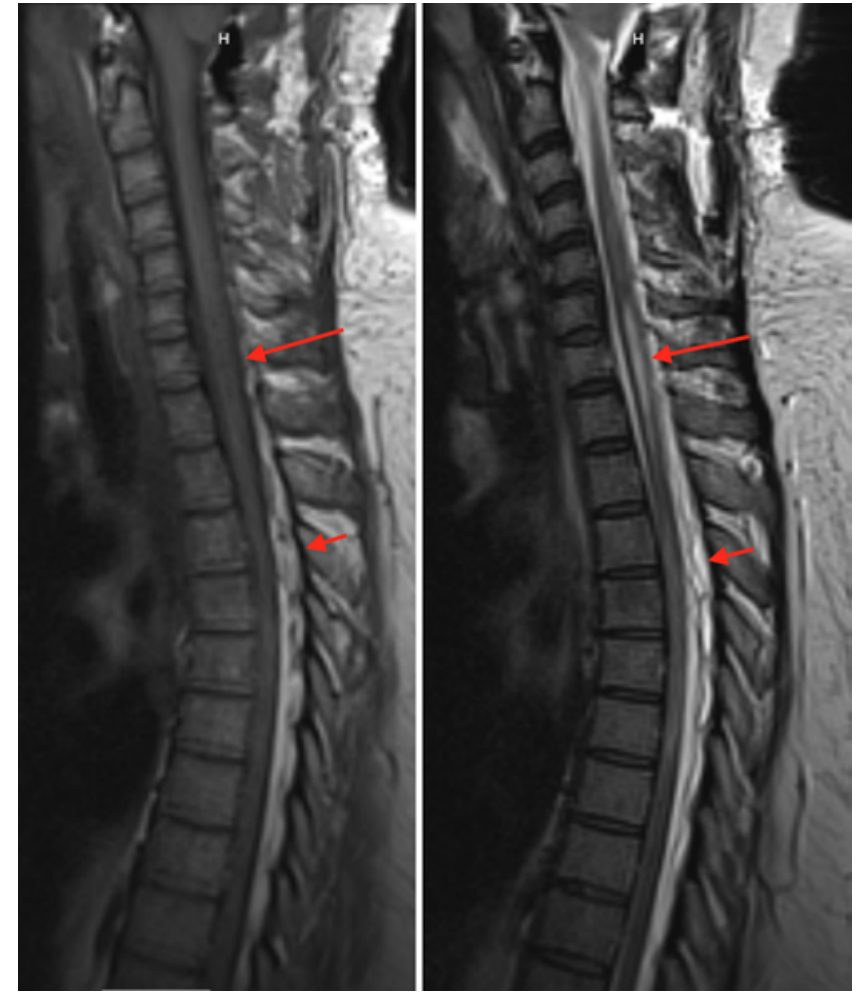

FIG. 4. Immediate postoperative sagittal T1- (left) and T2-weighted (right) images of the occipital-cervical and thoracic regions demonstrating evidence of the known syrinx (long red arrows) and equivocal findings of possible epidural hematoma at the site of the Chiari I decompression and in the upper thoracic region (short red arrows). Figure is available in color online only.

Spontaneous acute paresis has been documented in patients with syringomyelia associated with Chiari malformations. ${ }^{30,43}$ In these cases, however, symptoms resolved after surgical decompression. In one reported case, lowerextremity paresis was seen in the setting of cervical syrinx expansion due to shunt malfunction. ${ }^{4}$ Despite revision and adequate shunt function, the patient did not improve. Cases of neurological deterioration such as these suggest that the spinal cord is particularly vulnerable to vascular insults in individuals with a syrinx. After an extensive review of the literature, we identified no prior reports of paraplegia after Chiari decompression. Paraplegia has been described in a number of posterior fossa surgeries performed in patients in the prone or semisitting position,,$^{20,22,29,33,36,41}$ but these surgeries have been for posterior fossa tumors rather than Chiari malformations as in the patient reported here.

Preserving adequate spinal perfusion by maintaining sufficient mean arterial pressure, preventing hypercarbia, and avoiding excessive cervical flexion is necessary to prevent spinal cord injury in patients with normal body habitus, but these precautions alone may be insufficient for morbidly obese patients with a syrinx. The use of electrophysiological spinal cord monitoring modalities (such as somatosensory and/or motor evoked potentials) is not generally considered standard of care for Chiari decompressions. In fact, except for complex scoliosis and other
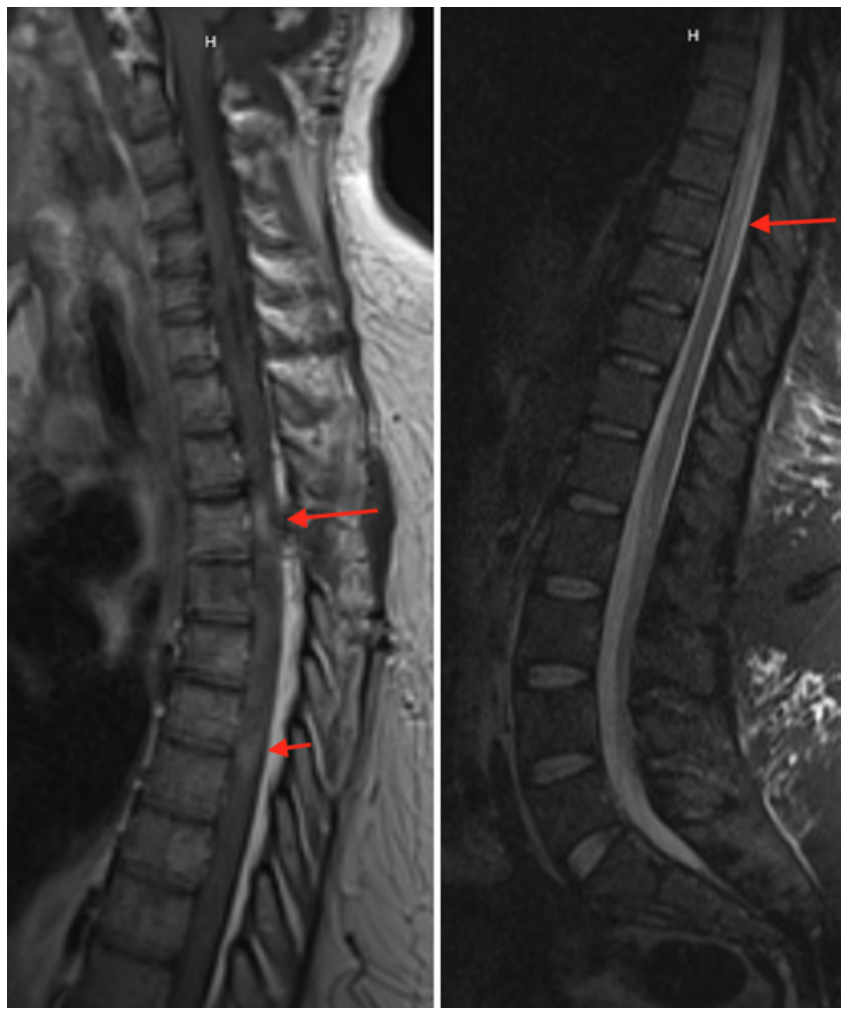

FIG. 5. Sagittal T1-weighted 1-week postoperative MR image with intravenous contrast of the occipital-cervical-thoracic region (left) and sagittal T2-weighted 1-week postoperative MR image of the thoracolumbar region (right) demonstrating evidence of the known syrinx (right, red arrow) with foci of increased enhancement and increased T2-signal abnormality in the mid- (left, long red arrow) and lower-thoracic (left, short red arrow) spinal cord, consistent with possible hyperemia and infarction. Figure is available in color online only.

spinal deformity surgeries, there is controversy as to the benefits of electrophysiological spinal cord monitoring. 2,39 Nonetheless, it is possible that electrophysiological spinal cord monitoring might alert the surgical and anesthesia teams of impending or early spinal cord injury. If such an "early warning" could successfully identify the onset of a decline in spinal cord function, then the spinal cord infarction could conceivably be avoided or reduced through modification of patient positioning, surgical technique, blood pressure, or other factors. Whether such measures would be effective is an issue of active debate, ${ }^{19,26}$ however, because if an immediate and irreversible acute spinal cord infarction occurs, actions taken after its onset may not improve the patient's outcome. For these reasons, some argue that the added costs and complexity that accompany routine application of electrophysiological spinal cord monitoring may not be justified. ${ }^{19}$

That said, the risks and costs of electrophysiological spinal cord monitoring are generally low, and thus spinal cord monitoring may be worth considering for higher-risk Chiari decompression surgeries. Along these lines, prophylactic insertion of a lumbar catheter for CSF drainage to decrease intraspinal pressure is used in thoracoabdominal aortic aneurysm surgery to reduce the risks of spinal 
cord injury. ${ }^{3}$ The placement of a lumbar drain involves challenges and risks, however, particularly in obese patients, and placement in a patient with low-lying tonsils may risk neurological consequences. Therefore, based on the current literature, there does not appear to be strong evidence to support the routine use of a lumbar drain during surgical decompression of Chiari malformations.

The occurrence of paraplegia after Chiari decompression is exceedingly rare and, to the authors' knowledge, has not been previously reported. Nevertheless, the neurosurgeon should remain cognizant of this potential complication, especially in cases with additional risk factors such as obesity, the presence of a syrinx, a potentially prolonged surgical procedure, or risk factors for vascular disease such as hypertension, diabetes mellitus, or tobacco use. The risk of paraplegia was likely increased for this obese patient with Chiari malformation and an associated syrinx who underwent surgery while in the prone position for an extended period of time. Standard precautions may be insufficient to prevent this complication in similar cases. Factors to consider include patient positioning (including the degree of neck flexion), cardiopulmonary dynamics, and the duration of the surgery. Application of intraoperative electrophysiological monitoring could be considered as an adjunct to potentially reduce unexpected neurological events.

\section{References}

1. Albayram S, Wasserman BA, Yousem DM, Wityk R: Intracranial hypotension as a cause of radiculopathy from cervical epidural venous engorgement: case report. AJNR Am J Neuroradiol 23:618-621, 2002

2. Anderson RCE, Dowling KC, Feldstein NA, Emerson RG: Chiari I malformation: potential role for intraoperative electrophysiologic monitoring. J Clin Neurophysiol 20:65-72, 2003

3. Augoustides JGT, Stone ME, Drenger B: Novel approaches to spinal cord protection during thoracoabdominal aortic interventions. Curr Opin Anaesthesiol 27:98-105, 2014

4. Beswick E, St George EJ, Solanki GA: Paraparesis secondary to expansion of cervical syrinx: an unusual presentation of ventriculo-peritoneal shunt malfunction. Pediatr Neurosurg 41:334-336, 2005

5. Bocca LF, Tonholo Silva TY, Ejnisman B, Pedroso JL, Barsottini OG: Teaching NeuroImages: The Charcot shoulder: Chiari malformation with syringomyelia associated with arthropathy. Neurology 89:e38-e39, 2017

6. Bond AE, Jane JA Sr, Liu KC, Oldfield EH: Changes in cerebrospinal fluid flow assessed using intraoperative MRI during posterior fossa decompression for Chiari malformation. J Neurosurg 122:1068-1075, 2015

7. Bowers CA, Brimley C, Cole C, Gluf W, Schmidt RH: AlloDerm for duraplasty in Chiari malformation: superior outcomes. Acta Neurochir (Wien) 157:507-511, 2015

8. Bracken MB, Shepard MJ, Holford TR, Leo-Summers L, Aldrich EF, Fazl M, et al: Administration of methylprednisolone for 24 or 48 hours or tirilazad mesylate for 48 hours in the treatment of acute spinal cord injury. Results of the Third National Acute Spinal Cord Injury Randomized Controlled Trial. National Acute Spinal Cord Injury Study. JAMA 277:1597-1604, 1997

9. Cesmebasi A, Loukas M, Hogan E, Kralovic S, Tubbs RS, Cohen-Gadol AA: The Chiari malformations: a review with emphasis on anatomical traits. Clin Anat 28:184-194, 2015

10. Chen J, Li Y, Wang T, Gao J, Xu J, Lai R, et al: Comparison of posterior fossa decompression with and without duraplasty for the surgical treatment of Chiari malformation type I in adult patients: a retrospective analysis of 103 patients. Medicine (Baltimore) 96:e5945, 2017

11. Clarot F, Callonnec F, Douvrin F, Hannequin D, Simonet J, Proust B, et al: Giant cervical epidural veins after lumbar puncture in a case of intracranial hypotension. AJNR Am J Neuroradiol 21:787-789, 2000

12. Cui LG, Jiang L, Zhang HB, Liu B, Wang JR, Jia JW, et al: Monitoring of cerebrospinal fluid flow by intraoperative ultrasound in patients with Chiari I malformation. Clin Neurol Neurosurg 113:173-176, 2011

13. Deng X, Wu L, Yang C, Xu Y: Neuropathic arthropathy caused by syringomyelia. J Neurosurg Spine 18:303-309, 2013

14. Dietemann JL, Bogorin A, Abu Eid M, Sanda R, Mourao Soares I, Draghici S, et al: Tips and traps in neurological imaging: imaging the perimedullary spaces. Diagn Interv Imaging 93:985-992, 2012

15. Edgcombe H, Carter K, Yarrow S: Anaesthesia in the prone position. Br J Anaesth 100:165-183, 2008

16. Everson RG, Holly LT, Batzdorf U: Chiari I malformation in the adult. Neurosurg Q 26:200-213, 2016

17. Foster E, Tsang BKT, Kam A, Storey E, Day B, Hill A: Hirayama disease. J Clin Neurosci 22:951-954, 2015

18. Freedman BA, Malone DG, Rasmussen PA, Cage JM, Benzel EC: Surfer's myelopathy: a rare form of spinal cord infarction in novice surfers: a systematic review. Neurosurgery 78:602-611, 2016

19. Hadley MN, Shank CD, Rozzelle CJ, Walters BC: Guidelines for the use of electrophysiological monitoring for surgery of the human spinal column and spinal cord. Neurosurgery 81:713-732, 2017

20. Hicdonmez T, Kilincer C, Hamamcioglu MK, Cobanoglu S: Paraplegia due to spinal subdural hematoma as a complication of posterior fossa surgery: case report and review of the literature. Clin Neurol Neurosurg 108:590-594, 2006

21. Hirabayashi Y, Shimizu R, Fukuda H, Saitoh K, Igarashi T: Soft tissue anatomy within the vertebral canal in pregnant women. Br J Anaesth 77:153-156, 1996

22. Hongo K, Nakagawa H, Tamai K, Kobayashi S: Paraplegia following surgery for medulloblastoma in the posterior fossa. J Clin Neurosci 6:522-524, 1999

23. Jiang E, Sha S, Yuan X, Zhu W, Jiang J, Ni H, et al: Comparison of clinical and radiographic outcomes for posterior fossa decompression with and without duraplasty for treatment of pediatric Chiari I malformation: a prospective study. World Neurosurg 110:e465-e 472, 2018

24. Langridge B, Phillips E, Choi D: Chiari malformation type 1: a systematic review of natural history and conservative management. World Neurosurg 104:213-219, 2017

25. Lee RR, Abraham RA, Quinn CB: Dynamic physiologic changes in lumbar CSF volume quantitatively measured by three-dimensional fast spin-echo MRI. Spine (Phila Pa 1976) 26:1172-1178, 2001

26. Li F, Gorji R, Allott G, Modes K, Lunn R, Yang ZJ: The usefulness of intraoperative neurophysiological monitoring in cervical spine surgery: a retrospective analysis of 200 consecutive patients. J Neurosurg Anesthesiol 24:185-190, 2012

27. Lin W, Duan G, Xie J, Shao J, Wang Z, Jiao B: Comparison of results between posterior fossa decompression with and without duraplasty for the surgical treatment of Chiari malformation type I: a systematic review and meta-analysis. World Neurosurg 110:460-474.e5, 2018

28. Lu VM, Phan K, Crowley SP, Daniels DJ: The addition of duraplasty to posterior fossa decompression in the surgical treatment of pediatric Chiari malformation Type I: a systematic review and meta-analysis of surgical and performance outcomes. J Neurosurg Pediatr 20:439-449, 2017 
29. Maduri R, Daniel RT, Diezi M, Cotting J, Messerer M: Paraplegia after posterior fossa surgery in prone position: can we prevent it? Childs Nerv Syst 33:25-26, 2017

30. Martínez-Lage JF, Alarcón F, López López-Guerrero A, Felipe-Murcia M, Ruíz-Espejo Vilar A, Almagro MJ: Syringomyelia with quadriparesis in CSF shunt malfunction: a case illustration. Childs Nerv Syst 26:1229-1231, 2010

31. McGirt MJ, Attenello FJ, Datoo G, Gathinji M, Atiba A, Weingart JD, et al: Intraoperative ultrasonography as a guide to patient selection for duraplasty after suboccipital decompression in children with Chiari malformation Type I. J Neurosurg Pediatr 2:52-57, 2008

32. Milhorat TH, Bolognese PA: Tailored operative technique for Chiari type I malformation using intraoperative color Doppler ultrasonography. Neurosurgery 53:899-906, 2003

33. Morandi X, Riffaud L, Amlashi SFA, Brassier G: Extensive spinal cord infarction after posterior fossa surgery in the sitting position: case report. Neurosurgery 54:1512-1516, 2004

34. Oldfield EH, Muraszko K, Shawker TH, Patronas NJ: Pathophysiology of syringomyelia associated with Chiari I malformation of the cerebellar tonsils. Implications for diagnosis and treatment. J Neurosurg 80:3-15, 1994

35. Parker SR, Harris P, Cummings TJ, George T, Fuchs H, Grant G: Complications following decompression of Chiari malformation Type I in children: dural graft or sealant? J Neurosurg Pediatr 8:177-183, 2011

36. Rau CS, Liang CL, Lui CC, Lee TC, Lu K: Quadriplegia in a patient who underwent posterior fossa surgery in the prone position. Case report. J Neurosurg 96 (1 Suppl):101-103, 2002

37. Rhoton AL Jr: Microsurgery of Arnold-Chiari malformation in adults with and without hydromyelia. J Neurosurg 45:473-483, 1976

38. Rocque BG, Oakes WJ: Surgical treatment of Chiari I malformation. Neurosurg Clin N Am 26:527-531, 2015

39. Roser F, Ebner FH, Liebsch M, Tatagiba MS, Naros G: The role of intraoperative neuromonitoring in adults with Chiari malformation. Clin Neurol Neurosurg 150:27-32, 2016

40. Saklad M: Grading of patients for surgical procedures. Anesthesiology 2:281-284, 1941
41. Salunke P, Gupta K, Kovai P, Sura S, Aggarwal A: Preoperative diffuse leptomeningeal spread in a medulloblastoma: paraplegia following surgery for posterior fossa and call for newer management protocols. J Pediatr Neurosci 6:152154,2011

42. Shinaver CN, Caldemeyer KS: Engorged anterior epidural venous plexus mimics an anterior epidural mass. J Neuroimaging 8:242-244, 1998

43. Vlcek BW, Ito B: Acute paraparesis secondary to ArnoldChiari type I malformation and neck hyperflexion. Ann Neurol 21:100-101, 1987

44. Xu H, Chu L, He R, Ge C, Lei T: Posterior fossa decompression with and without duraplasty for the treatment of Chiari malformation type I-a systematic review and meta-analysis. Neurosurg Rev 40:213-221, 2017

45. Zhao JL, Li MH, Wang CL, Meng W: A systematic review of Chiari I malformation: techniques and outcomes. World Neurosurg 88:7-14, 2016

\section{Disclosures}

Dr. Chicoine reports receiving clinical or research support for the study described (includes equipment or material) from IMRIS.

\section{Author Contributions}

Conception and design: Yahanda, Shah, Chicoine. Acquisition of data: Shah, Chicoine. Analysis and interpretation of data: Shah, Athiraman, Tempelhoff, Chicoine. Drafting the article: all authors. Critically revising the article: all authors. Reviewed submitted version of manuscript: Yahanda, Shah, Chicoine. Approved the final version of the manuscript on behalf of all authors: Yahanda. Study supervision: Chicoine.

\section{Correspondence}

Alexander T. Yahanda: Department of Neurosurgery, Washington University School of Medicine, St. Louis, MO. ayahanda@wustl. edu. 